\title{
SPHK1 deficiency protects mice from acetaminophen-induced ER stress and mitochondrial permeability transition
}

\author{
Longjun $\mathrm{Li}^{1} \cdot$ Haitao Wang ${ }^{1}$ Jun $\mathrm{Zhang}^{1} \cdot$ Yeqin Sha ${ }^{1} \cdot$ Fengjiao $\mathrm{Wu}^{1} \cdot$ Shuang Wen ${ }^{1} \cdot$ Lianping He ${ }^{1}$ Liang Sheng ${ }^{2}$.

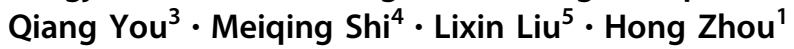

Received: 9 December 2018 / Revised: 25 November 2019 / Accepted: 27 November 2019 / Published online: 11 December 2019

(c) The Author(s), under exclusive licence to ADMC Associazione Differenziamento e Morte Cellulare 2019

\begin{abstract}
Acetaminophen (APAP) is the leading cause of drug-induced acute liver failure. Sphingosine-1-phosphate (S1P), whose formation is catalyzed by sphingosine kinase (SPHK)-1 or -2, is a bioactive lipid implicated in human health and disease. Here, we show that APAP-treated $s p h K 1$-deficient $\left(s p h \mathrm{Kl}^{-/-}\right)$mice exhibited markedly less liver damage and reduced inflammation compared with the wild-type mice. SPHK1 deficiency alleviated APAP-induced endoplasmic reticulum (ER) stress by affecting the phosphorylation of inositol-requiring enzyme $1 \alpha(\mathrm{IRE} 1 \alpha)$ and protein kinase R (PKR)-like endoplasmic reticulum kinase (PERK)-eukaryotic translation initiation factor $2 \alpha(\mathrm{eIF} 2 \alpha)$, levels of activating transcription factor 4 (ATF4), and activation of activating transcription factor 6 (ATF6). SPHK1 deficiency also inhibited mitochondrial permeability transition (MPT), as evidenced by the impaired phosphorylation of JNK, apoptosis signal-regulated kinase 1 (ASK1), and glycogen synthase kinase $3 \beta$ (GSK3 $\beta$ ). In addition, SPHK1 deficiency reduced the levels of histone deacetylase and promoted the acetylation of p65 and STAT1, thereby impairing the transcription of inflammatory genes. Supplementation with exogenous S1P significantly reversed the activation of the PERK-eIF2 $\alpha$-ATF4 pathway and ATF6 during ER stress as well as the activation of GSK3 $\beta$, ASK1, and JNK during MPT. Both FTY720, a functional S1P receptor antagonist, and PF543, an SPHK1 inhibitor, significantly ameliorated APAP-induced liver injury and improved animal survival. Our study reveals a critical role for SPHK1 in mediating APAP-induced hepatotoxicity by promoting ER stress and MPT.
\end{abstract}

These authors contributed equally: Longjun Li, Haitao Wang

Edited by G. Del Sal

Supplementary information The online version of this article (https:// doi.org/10.1038/s41418-019-0471-x) contains supplementary material, which is available to authorized users.

Hong Zhou

hzhou@njmu.edu.cn

1 Department of Immunology, Nanjing Medical University, Nanjing101 Longmian Avenue, Jiangsu 211166, China

2 Department of Pharmacology, Nanjing Medical University, Nanjing101 Longmian Avenue, Jiangsu 211166, China

3 Department of Biotherapy, Second Affiliated Hospital, Nanjing Medical University, Nanjing121 Jiangjiayuan Road, Jiangsu 210011, China

4 Division of Immunology, Virginia-Maryland College of Veterinary Medicine, University of Maryland, College Park, MD 20742, USA

5 Department of Anatomy, Physiology and Pharmacology, University of Saskatchewan, Saskatoon, Saskatchewan S7N 5E5, Canada

\section{Introduction}

Acetaminophen (APAP), a common antipyretic and analgesic, is currently the most common cause of druginduced liver injury following abuse or accidental overdose. APAP-induced liver injury accounts for $>10,000$ hospital visits and $\sim 500$ deaths in the United States annually [1-3]. Numerous studies have shown that the toxicity is primarily due to a reactive APAP metabolite, $N$-acetyl- $p$-benzoquinone imine (NAPQI), which rapidly depletes glutathione (GSH), binds to mitochondrial membrane proteins, interferes with mitochondrial functions and ATP production, induces nuclear DNA damage, and elicits massive necrosis [4-6]. Hepatocyte necrosis activates Kupffer cells and recruits peripheral immune cells, further aggravating hepatocyte injury and inflammation [7-9].

In hepatocytes, the endoplasmic reticulum (ER) is the major site of protein synthesis, folding, and maturation, as well as triglyceride synthesis and drug metabolism. In APAPtreated hepatocytes, ER stress can be initiated when the influx of unfolded or misfolded proteins exceeds the folding 
capacity of the ER, triggering cellular necrosis if excessively severe or prolonged. The unfolded protein response is mediated by three distinct sensors in the ER: inositolrequiring enzyme $1 \alpha$ (IRE1 $\alpha$ ), activating transcription factor 6 (ATF6), and protein kinase R (PKR)-like endoplasmic reticulum kinase (PERK) [10-14]. In turn, IRE1 $\alpha$ and PERK-eIF $2 \alpha$ activate C/EBP-homologous protein (CHOP), a protein that translocates to the nucleus and activates the BCL-2 family member BAX and promotes oxidative stress and inflammation [15]. In addition, IRE1 $\alpha$ activates c-Jun N-terminal kinase (JNK), which then forms a tripartite complex with apoptosis signal-regulated kinase 1 (ASK1) and tumor necrosis factor (TNF) receptorassociated factor 2 (TRAF2) to induce the expression of inflammatory genes $[16,17]$. Acting as a critical threshold of mitochondrial stress, JNK plays an important role in the stress response and induces the mitochondrial permeability transition (MPT), subsequently compromising ATP production and eliciting necrotic cell death [18-22].

Sphingosine-1-phosphate (S1P), a bioactive lipid that has been implicated in human health and disease, regulates cell migration, angiogenesis, inflammation, and immune cell trafficking via its receptors, S1PR1-5 [23-25]. S1P is produced by the phosphorylation of sphingosine, a reaction catalyzed by sphingosine kinase 1 or 2 (SPHK1 or SPHK2). Both SPHK1 and SPHK2 are abundantly expressed in the liver [26, 27]. Intriguingly, SPHK1 induces epithelial-mesenchymal transition by promoting the autophagy-linked lysosomal degradation of CDH1/E-cadherin in hepatoma cells [28]. In addition, SPHK1 promotes hepatocellular migration and invasion via the S1P/EDG1 axis. Pharmacological inhibition of SPHK1 activity has been shown to inhibit liver tumorigenesis in diethylnitrosamine-treated mice. However, the role of sphingosine signaling in drug-induced liver injury is unknown. We now report that ER stress, MPT, and histone acetylation are

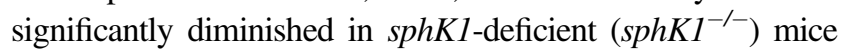
exposed to APAP, highlighting a critical role for SPHK1 in acute liver damage. Therefore, targeting SPHK1 may provide a novel strategy for treating APAP-induced hepatotoxicity.

\section{Results}

\section{Loss of SPHK1 protects hepatocytes from APAP- induced hepatotoxicity and inflammation}

Both SPHK1 and SPHK2 were highly expressed in the WT mouse liver (Fig. 1a). APAP treatment substantially increased the levels of SPHK1 in the liver at 2, 4, 8, and $16 \mathrm{~h}$, whereas SPHK2 levels remained unchanged (Fig. 1a). In primary hepatocytes from WT mice, treatment with APAP significantly enhanced levels of SPHK1 but not SPHK2 protein at 2 and $4 \mathrm{~h}$ (Fig. 1a). We next examined the levels of SPHK2 in $s p h \mathrm{Kl}^{-/-}$mice to determine whether the expression of SPHK2 would be upregulated to compensate for the absence of SPHK1 activity. Following APAP treatment, there was no significant change in SPHK2 protein levels in the livers of either WT or $s p h \mathrm{Kl}^{-/-}$mice (Fig. S1). We next evaluated the functional role of SPHK1 in APAP-induced hepatotoxicity by comparing $s p h \mathrm{Kl}^{-/-}$with WT mice. When treated with APAP at different doses and time points, the serum levels of alanine aminotransferase (ALT) and aspartate aminotransferase (AST) were significantly lower in $s p h K^{-/-}$mice than in WT mice (Fig. 1b), whereas the levels of these enzymes in $s p h K^{-/-}$mice were identical to those in WT mice (Fig. S2). Moreover, serum levels of IL- 6 , TNF- $\alpha$, and IL- $1 \beta$ at $16 \mathrm{~h}$ post APAP treatment were also significantly lower in $s p h K 1^{-/-}$mice (Fig. 1c). Importantly, sphK1 ${ }^{-/-}$mice had a much higher survival rate than WT mice when treated with a lethal dose $(700 \mathrm{mg} / \mathrm{kg}$ ) of APAP (Fig. 1d). There was also much less extensive centrilobular necrosis at 8 and $16 \mathrm{~h}$ following APAP treatment in $s p h K 1^{-1-}$ mice than in WT mice, as assessed by H\&E staining (Fig. 1e). Furthermore, terminal deoxynucleotidyl transferase dUTP nick end labeling (TUNEL) of liver sections (Fig. 1f) and propidium iodide staining (Fig. S3) also revealed a reduction in hepatocyte necrosis in $\operatorname{sph} \mathrm{KI}^{-/-}$mice when compared to that in WT mice at $16 \mathrm{~h}$ post APAP exposure. PF543, an inhibitor of SPHK1, significantly reduced the elevated serum levels of ALT and AST (Fig. 1g) and improved the survival rate in WT mice treated with APAP (Fig. 1h). Collectively, these data indicate that SPHK1 has an essential role in the development of APAP-induced liver injury.

APAP exposure substantially induced the infiltration of Ly- $6 \mathrm{G}^{+}$neutrophils (Fig. 2a) and $\mathrm{F} 4 / 80^{+}$macrophages (Fig. 2b) into hepatic tissues in WT mice, but not in $s p h \mathrm{Kl}^{-/-}$mice (Fig. 2a, b). Moreover, APAP exposure substantially enhanced leukocyte recruitment into the liver

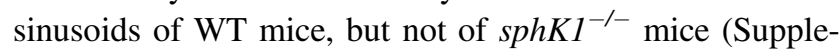
mentary Video 1). APAP treatment also boosted the expression levels of $I l-6, T n f-\alpha$, and $I l-1 \beta$ mRNAs in WT mice at $16 \mathrm{~h}$ post APAP administration. However, the increases in the expression levels of the mRNAs encoding these inflammatory cytokines were significantly lower in $s p h K 1^{-1-}$ (Fig. 2c), but not in $s p h K 2^{-/-}$mice (Fig. S4a). Similar trends were observed for the mRNA levels of inflammatory chemokines, such as $\mathrm{Ccl} 2, \mathrm{Ccl} 3, \mathrm{Cxcll}$, and Cxcl2 (Fig. 2d, Fig. S4b). Collectively, the above observations indicate that SPHK1 deficiency mitigates the liver injury and inflammation induced by APAP treatment.

\section{SPHK1 in resident hepatic cells mediates APAP- induced liver damage and inflammation}

To determine whether APAP-induced hepatotoxicity depends on SPHK1 activities in resident hepatic cells or 
A
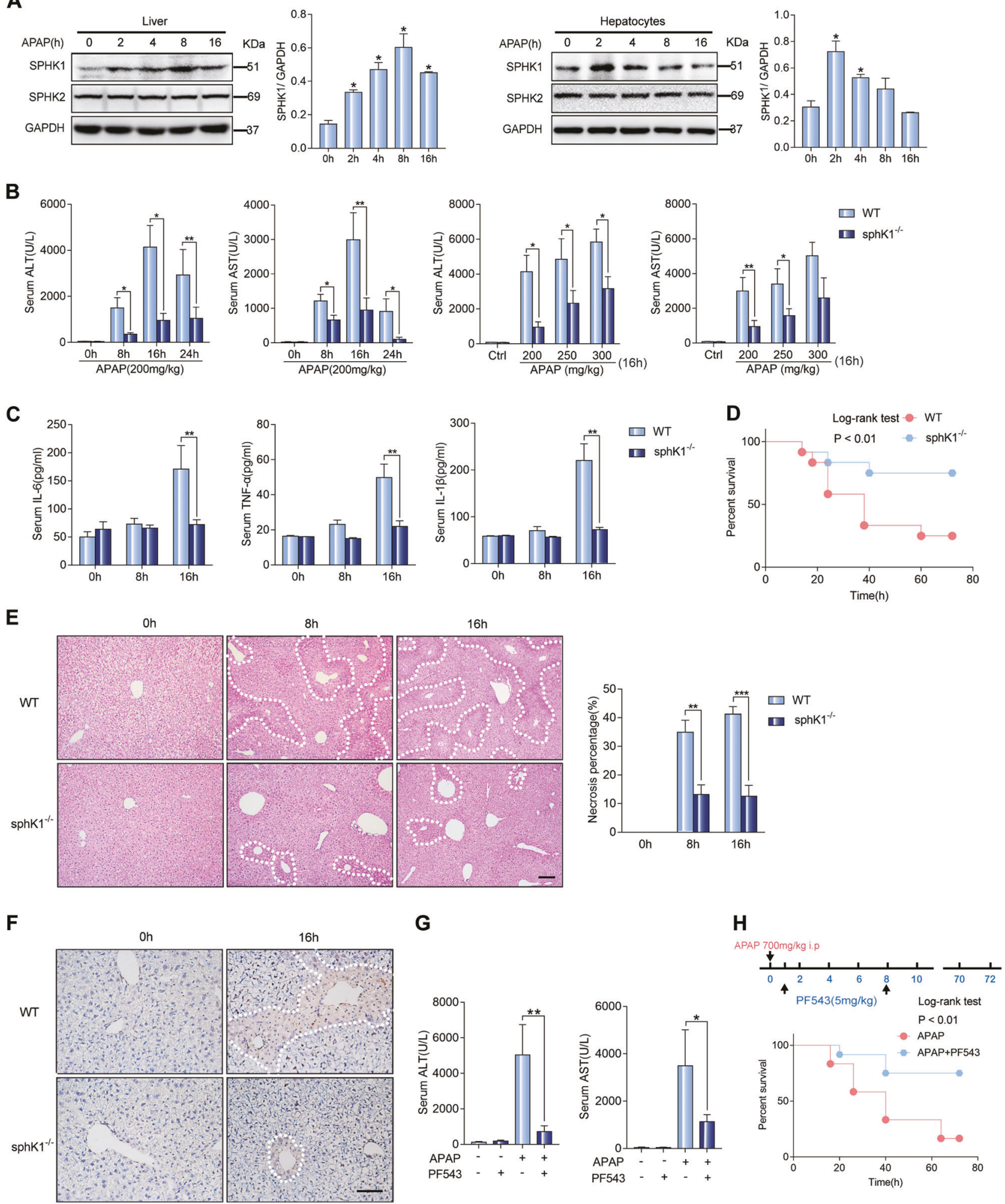

infiltrating immune cells, we generated chimeric mice by transferring bone marrow cells between WT and $s p h \mathrm{~K}^{-/-}$ mice (Fig. 3a). Remarkably, when exposed to APAP, $s p h \mathrm{~K}^{-1-}$ mice engrafted with WT bone marrow displayed a much lower ALT and AST serum levels than WT control mice or WT mice engrafted with either $s p h K 1^{-1-}$ or WT 
Fig. 1 APAP treatment reduces liver injury and hepatocyte necrosis in $\boldsymbol{s p h K 1 ^ { - 1 - }}$ mice. a SPHK1 and SPHK2 protein levels determined by western blotting in liver tissue of wild-type (WT) mice treated with APAP $(200 \mathrm{mg} / \mathrm{kg}$, i.p. $)$ and in cultured primary WT hepatocytes treated with APAP $(10 \mathrm{~mm})$. b WT and $s p h K 1^{-/-}$mice were treated with APAP $(200,250,300 \mathrm{mg} / \mathrm{kg}$, i.p., $n=6-8$ per group $)$, and then sera were harvested at $0,8,16$, and $24 \mathrm{~h}$ post APAP treatment. ALT and AST were assayed by commercial kits. $\mathbf{c}$ WT and $s p h K 1^{-1-}$ mice were treated with APAP $(200 \mathrm{mg} / \mathrm{kg}$, i.p., $n=6-8$ per group $)$, and serum levels of inflammatory cytokines IL- 6 , TNF- $\alpha$, and IL- $1 \beta$ were determined by ELISA. d Survival curve of WT and $\operatorname{sphK1^{-\prime -}}$ mice following treatment of a lethal dose of APAP $(700 \mathrm{mg} / \mathrm{kg}$, i.p., $n=12$ per group). e H\&E staining and quantification of necrotic areas (circled with white dots) in liver sections from WT and $s p h K 1^{-1-}$ mice treated with APAP ( $200 \mathrm{mg} / \mathrm{kg}$, i.p.). Scale bar: $200 \mu \mathrm{m}$. Data represent the mean ratio of the necrotic area to the total area \pm SEM. $\mathbf{f}$ WT and

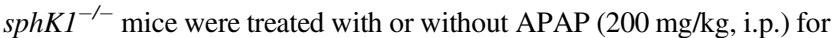
$16 \mathrm{~h}$. TUNEL staining was performed on liver sections; scale bar: $100 \mu \mathrm{m}$. g WT mice were treated with APAP $(200 \mathrm{mg} / \mathrm{kg}$, i.p. $)$ or with APAP and PF543 $(10 \mathrm{mg} / \mathrm{kg}$, i.p., $n=6$ per group), and serum levels of ALT and AST were determined at $16 \mathrm{~h}$ post APAP treatment. h Survival curve of WT mice treated with a lethal dose of APAP $(700 \mathrm{mg} / \mathrm{kg}$, i.p.) and of WT mice treated with APAP and PF543 $(5 \mathrm{mg} / \mathrm{kg}$, i.p., $n=12$ per group). $* P<0.05 ; * * P<0.01 ; * * * P<0.001$ using twotailed unpaired Student's $t$ test.

bone marrow (Fig. 3b). In addition, Il-6, Tnf- $\alpha, I l-1 \beta$, and Ccl2 mRNA levels in hepatic tissue from WT mice

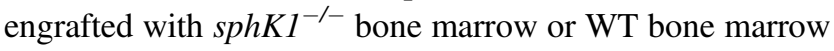
were much higher at $16 \mathrm{~h}$ post APAP injection than those in

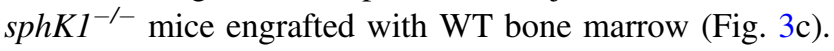
Furthermore, the severity of centrilobular necrosis was

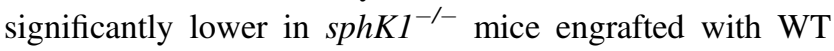
bone marrow than in WT mice engrafted with either sphK1 $1^{-/-}$or WT bone marrow (Fig. 3d). Together, these results indicate that SPHK1 in bone marrow-derived cells does not contribute to the development of acute inflammatory liver injury. Therefore, it is likely that the SPHK1 in resident hepatic cells mediates APAP-induced acute liver injury and inflammation.

\section{SPHK1 deficiency reduces APAP-induced JNK activation and hepatocyte death}

Having demonstrated an essential role for SPHK1 in resident hepatic cells in APAP-induced liver injury, we next isolated hepatocytes and further investigated the molecular signaling mechanisms underlying the role of SPHK1 in promoting hepatic cell death. In primary cultures of WT hepatocytes, exposure to APAP for $8 \mathrm{~h}$ induced a significant increase in cell death, as evidenced by staining with propidium iodide (Fig. 4a). However, the number of necrotic cells was markedly decreased in pri-

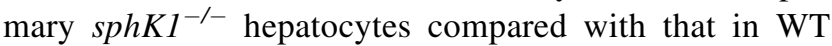
hepatocytes (Fig. 4a). When mice were treated with APAP, JNK phosphorylation in the liver was significantly increased in WT mice at 8-16 h, but not in $s p h \mathrm{~K}^{-/-}$mice (Fig. 4b). Similarly, the levels of phosphorylated JNK

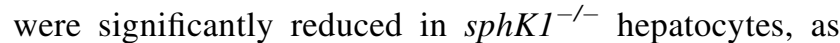
compared with those in hepatocytes from WT mice (Fig. 4c). The levels of BAX were increased in WT hepatocytes $2-8 \mathrm{~h}$ post APAP exposure, but not in sphK1 $1^{-/-}$hepatocytes. However, BCL-2 levels were comparable between WT and $\operatorname{sphK1^{-1-}}$ cells (Fig. 4d). Furthermore, SPHK1 deficiency resulted in reduced levels of phosphorylated ASK1 (Fig. S5a) and GSK3 $\beta$ (Fig. S5b) compared with those in WT hepatocytes. The above results indicate that SPHK1 deficiency protects hepatocytes against APAP-induced necrotic cell death by alleviating sustained JNK activation.

\section{SPHK1 deficiency alleviates ER stress}

APAP is metabolized in the liver by the cytochrome P450 isozyme CYP2E1 into NAPQI, which is then detoxified by hepatocellular GSH. When challenged with an overdose of APAP, SPHK1 deficiency did not result in any change in the levels of CYP2E1 (Fig. 5a) or GSH (Fig. 5b) in the liver of $s p h \mathrm{Kl}^{-1-}$ mice, as compared with those in WT mice. Therefore, the resistance of $\operatorname{sphK1^{-1-}}$ mice to APAPinduced liver injury cannot be attributed to an increase in APAP metabolism.

ER stress occurs following APAP administration, and multiple molecules including CHOP play an essential role in APAP-induced hepatotoxicity [10]. We then examined whether SPHK1 deficiency affected signal activation upon ER stress. There were no differences in the basal levels of

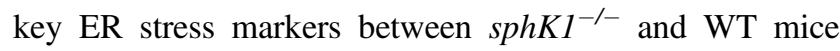
after an overnight fast. CHOP levels were markedly increased in WT mice upon APAP exposure. However, in $s p h \mathrm{Kl}^{-/-}$mice, this increase was completely abolished (Fig. 5c). Moreover, the activation of IRE1 $\alpha$ was severely affected by SPHK1 deficiency (Fig. 5d), resulting in reduced expression of $\mathrm{Xbpl}$ (s/t) (Fig S6a), and a decreased amount of IRE1-dependent decay of mRNA (RIDD) (Fig. S6b). The activation of the PERK-eIF2 $\alpha$-ATF4 pathway was also severely affected by SPHK1 deficiency (Fig. 5e). Similarly, the levels of uncleaved and cleaved ATF6 were increased in a time-dependent manner upon APAP stimulation in WT, but not in $s p h \mathrm{Kl}^{-/-}$mice (Fig. 5f). Further, we examined the expression of ATF6's target genes to evaluate ATF6 activation and observed significant upregulation of HerpUD and Hyoul in the liver of WT mice treated with APAP, but not in the liver of $s p h \mathrm{Kl}^{-/-}$mice (Fig. 5g). In addition, silencing the expression of IREI-a, Perk, or Eif $2 a$ reduced APAPinduced hepatocyte death (Fig. 5h). In primary hepatocytes treated with APAP, the levels of CYP2E1 in $s p h \mathrm{K1}^{-/-}$ primary hepatocytes were comparable to those in WT primary hepatocytes after APAP treatment (Fig. S7a). However, the activation of molecules mediating ER stress was 
A

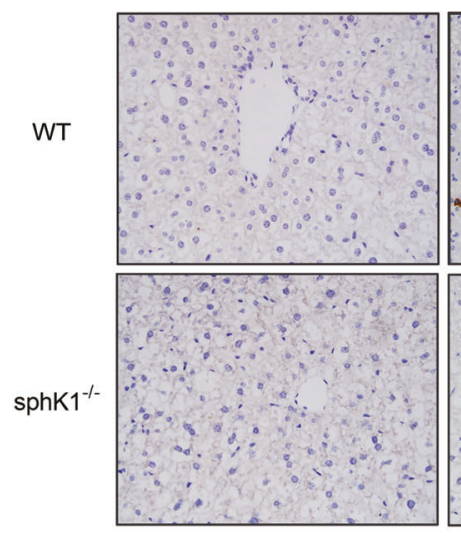

$8 \mathrm{~h}$

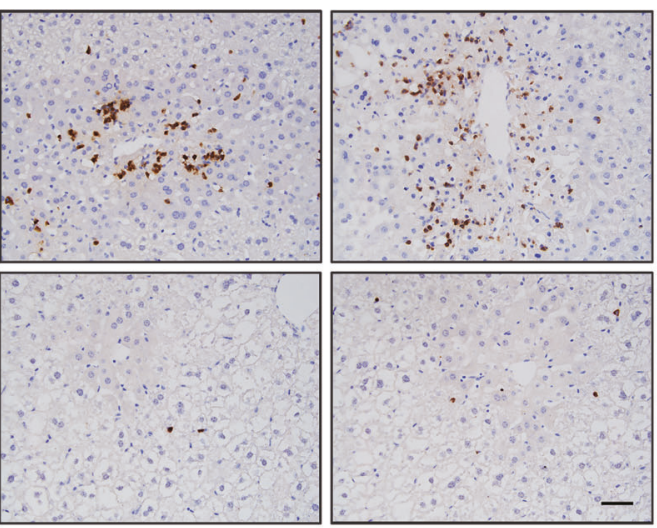

B

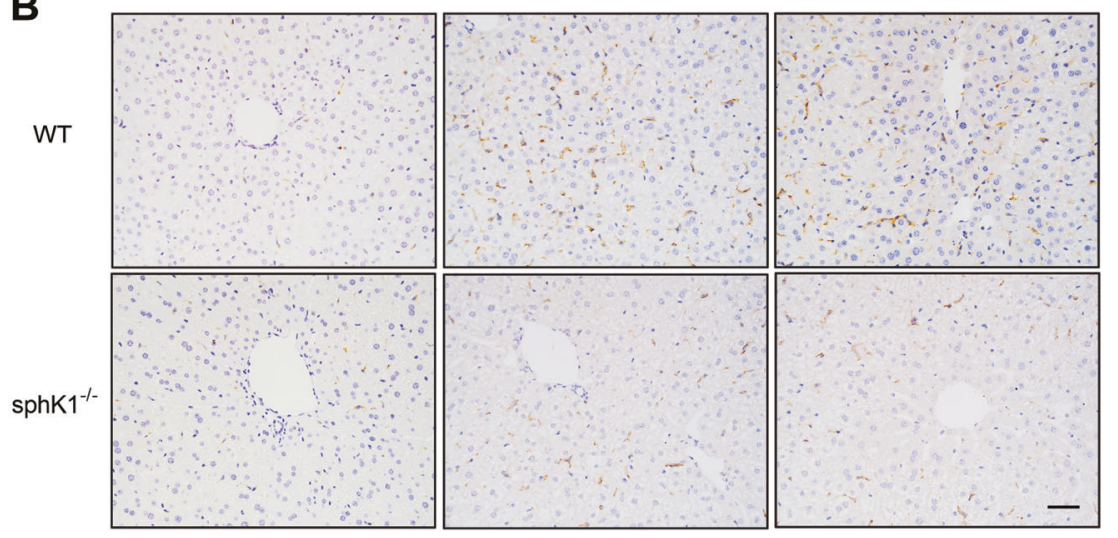

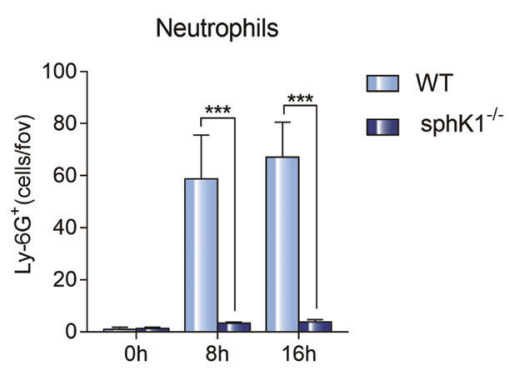

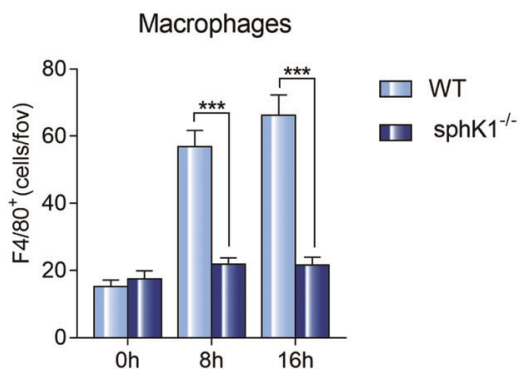

C

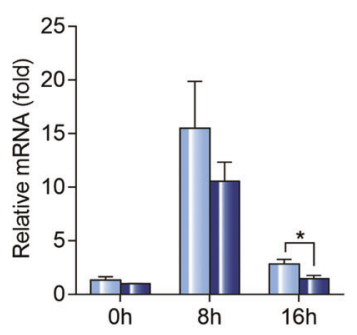

D

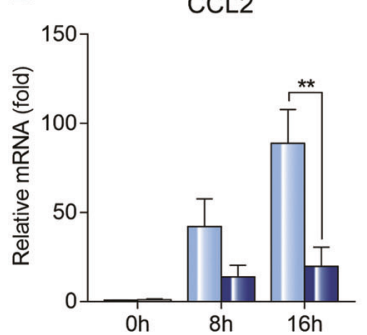

TNF-a

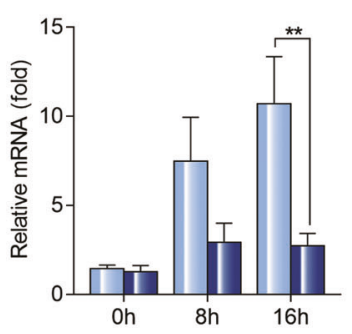

CCL3

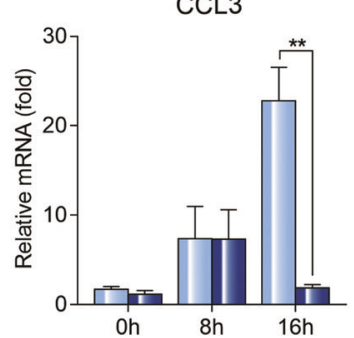

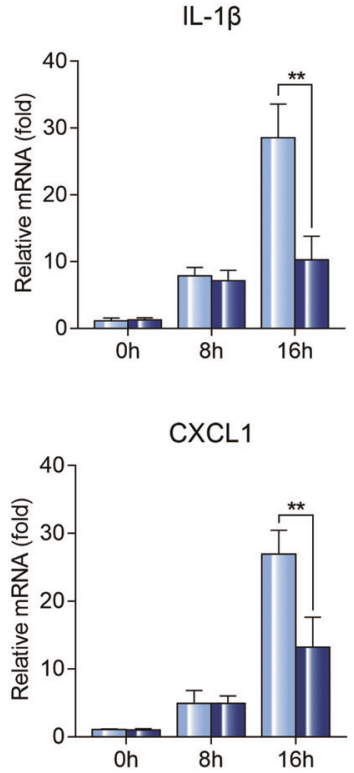

WT

sphK1 $1^{-1-}$

Fig. 2 APAP-treated $\operatorname{sphK1^{-/-}}$ mice display reduced liver inflammation and leukocyte recruitment. a, b WT or $s p h \mathrm{~K}^{-/-}$mice were treated with APAP $(200 \mathrm{mg} / \mathrm{kg}$, i.p. $)$, and then infiltrated a neutrophils $\left(\mathrm{Ly}-6 \mathrm{G}^{+}\right)$and $\mathbf{b}$ macrophages $\left(\mathrm{F} 4 / 80^{+}\right)$in liver tissues were quantified at 0,8 , and $16 \mathrm{~h}$ after APAP treatment. Scale bar: $100 \mu \mathrm{m}$; per field of view (fov, $n=8$ per group). c, d mRNA levels of $\mathbf{c}$ inflammatory cytokines Il-6, Tnf- $\alpha$, and $I l-1 \beta$ and $\mathbf{d}$ chemokines $C c l 2, C c l 3, C x c l 1$, and $C x c l 2$ in liver tissues of WT and $\operatorname{sphK1^{-/-}}$ mice at 0,8 , and $16 \mathrm{~h}$ after treatment with APAP $(200 \mathrm{mg} / \mathrm{kg}$, i.p.) were measured by quantitative real-time PCR. Data represent mean relative expression \pm SEM. $* P<0.05 ; * * P<0.01 ; * * * P<0.001$ using two-tailed unpaired Student's $t$ test. 
A

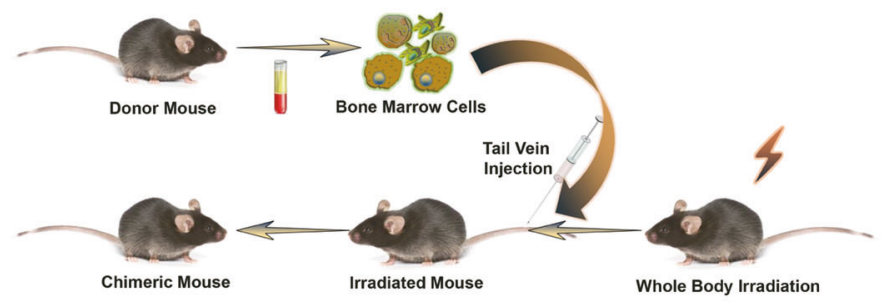

C

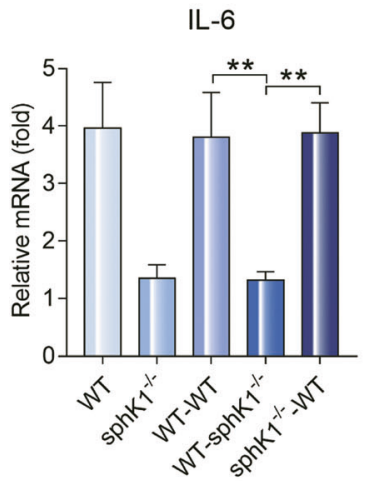

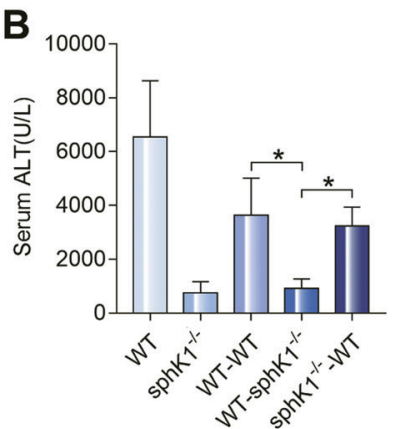

IL-1 $\beta$
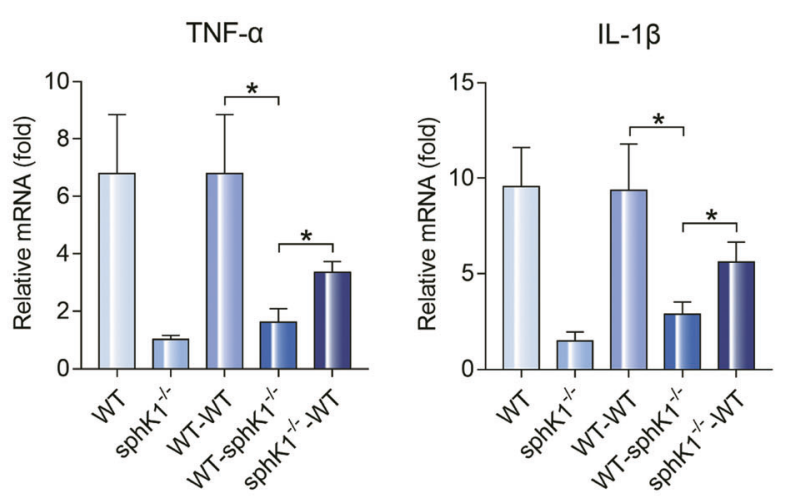
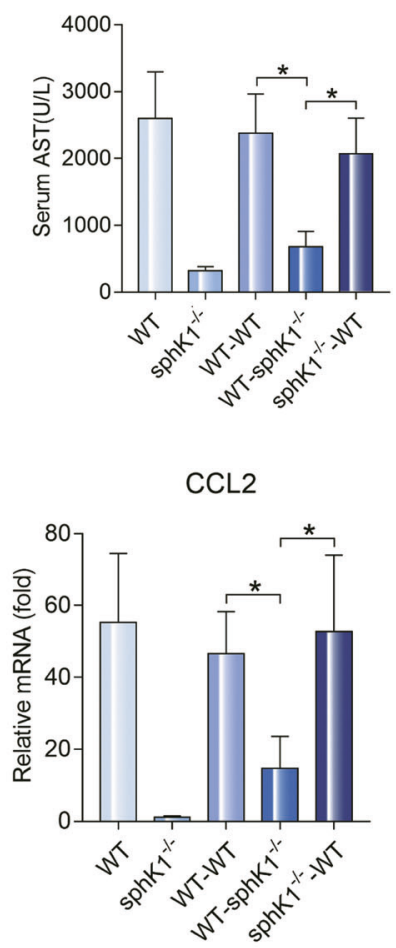

D
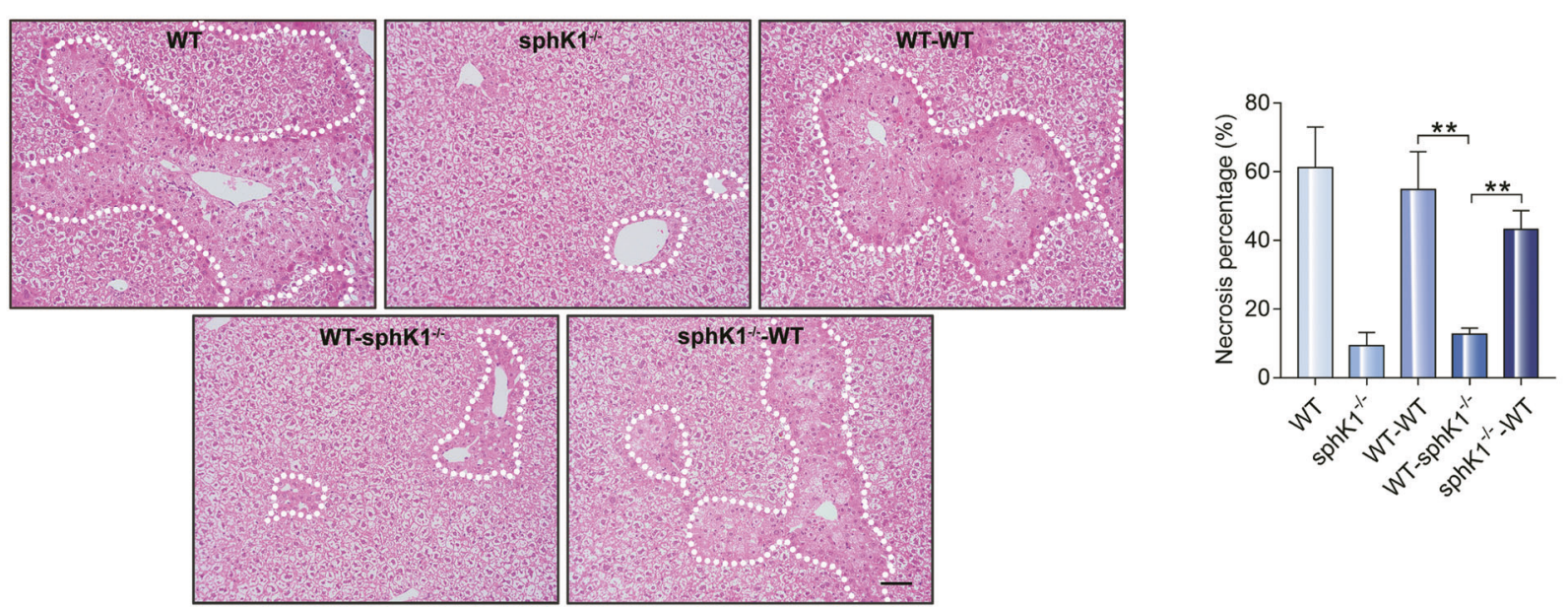

Fig. 3 SPHK1 in residential hepatic cells mediates liver injury and inflammation after APAP treatment. a Schematic protocol used for bone marrow transplantation between WT and $s p h \mathrm{KI}^{-/-}$mice. $\mathbf{b}-\mathbf{d}$ Nontransplanted WT and $s p h K 1^{-/-}$mice and WT mice engrafted with WT and $\operatorname{sphK1^{-/-}}$ bone marrow (designated as WT-WT and $\operatorname{sphK1^{-/}-\mathrm {WT}}$ chimeric mice, respectively), or $\operatorname{sphK1^{-/}}$ mice engrafted with WT bone marrow (designated as WT-sphK1 ${ }^{-1-}$ chimeric mice) were treated with
APAP (200 mg/kg, $n=8$ per group), b and serum levels of ALT and AST were quantified by commercial kits $16 \mathrm{~h}$ post APAP treatment. c mRNA levels of $I l-6, T n f-\alpha, I l-1 \beta$, and $C c l 2$ and $\mathbf{d}$ necrotic area in liver tissue of chimeric mice were quantified by real-time PCR and H\&E staining, respectively. Scale bar: $200 \mu \mathrm{m}$. Data represent the mean ratio of the necrotic area to the total area \pm SEM. $* P<0.05 ; * * P<0.01$ using two-tailed unpaired Student's $t$ test. similarly affected by SPHK1 deficiency (Fig. S7b-e). Collectively, these data imply that SPHK1 is not involved in APAP metabolism but instead promotes ER stress in response to the presence of hepatotoxic metabolites that are formed following APAP treatment.

Treatment with tunicamycin (TUN) and thapsigargin (TG) enhanced the activation of PERK-eIF $2 \alpha-$ ATF4 and ATF6 as well as the phosphorylation of IRE1 $\alpha$ in WT but

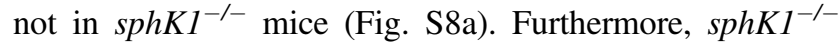
mice exhibited a significantly higher survival rate than did WT mice after treatment with a lethal dose of TG (Fig. S8b). Therefore, SPHK1 deficiency also dampens UPR activation and cell death in response to other agents that trigger ER stress, and the protection provided by SPHK1 deficiency is not only limited to the hepatotoxic effects induced by APAP. 


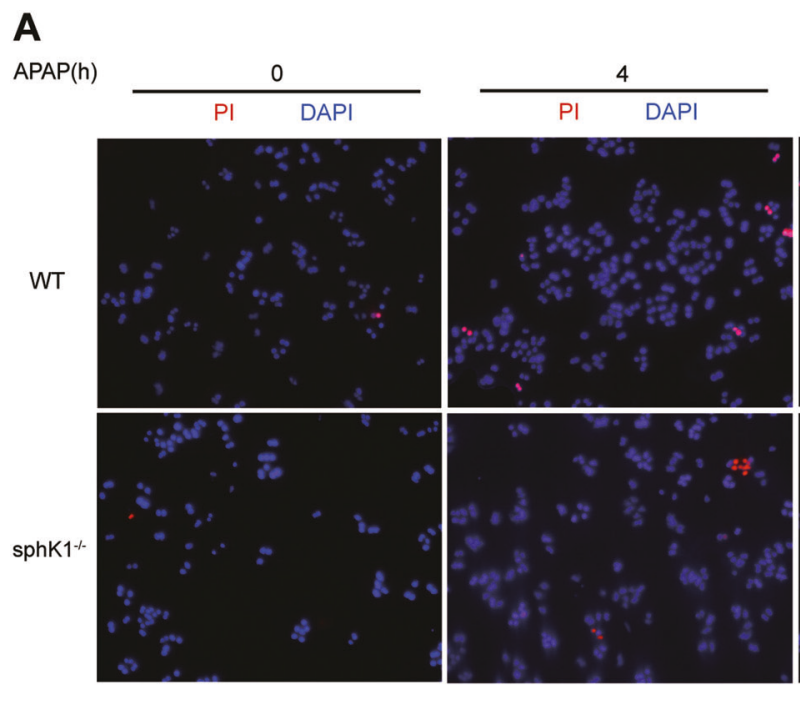

B

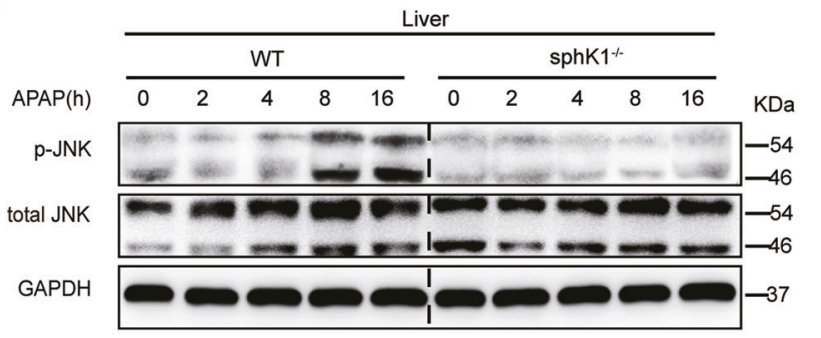

D

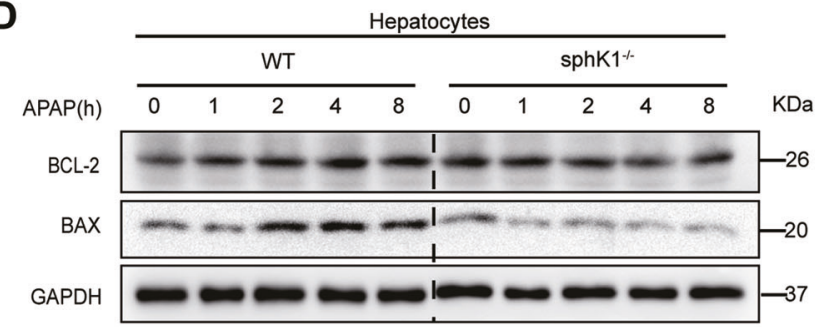

Fig. 4 SPHK1 deficiency attenuates JNK activation and protects hepatocytes from APAP-induced cell death. a Primary hepatocytes were isolated from WT or $s p h K 1^{-/}$mice and were treated with $10 \mathrm{~mm}$ APAP for $4 \mathrm{~h}$ or $8 \mathrm{~h}$. Dead cells were stained with propidium iodide (PI), and the percentage of necrotic cells was quantified. Data represent relative expression \pm SEM. $* * * P<0.001$ using two-tailed unpaired Student's $t$ test. b WT and $\operatorname{sphK1^{-1}}$ mice were treated with APAP

\section{APAP does not compromise mitochondrial function in the absence of SPHK1}

APAP-induced hepatotoxicity is characterized by the extensive production of ROS during drug metabolism. Mitochondrial ROS levels were increased in WT hepatocytes at 4-8 h of APAP exposure. However, in the absence of SPHK1, the mitochondrial accumulation of ROS was significantly reduced in APAP-treated hepatocytes (Fig. 6a). Moreover, upon APAP treatment, SPHK1 deficiency resulted in reduced JNK (Fig. 6b), ASK1 (Fig. 6c), and GSK3 $\beta$ (Fig. 6d) phosphorylation levels in mitochondria.
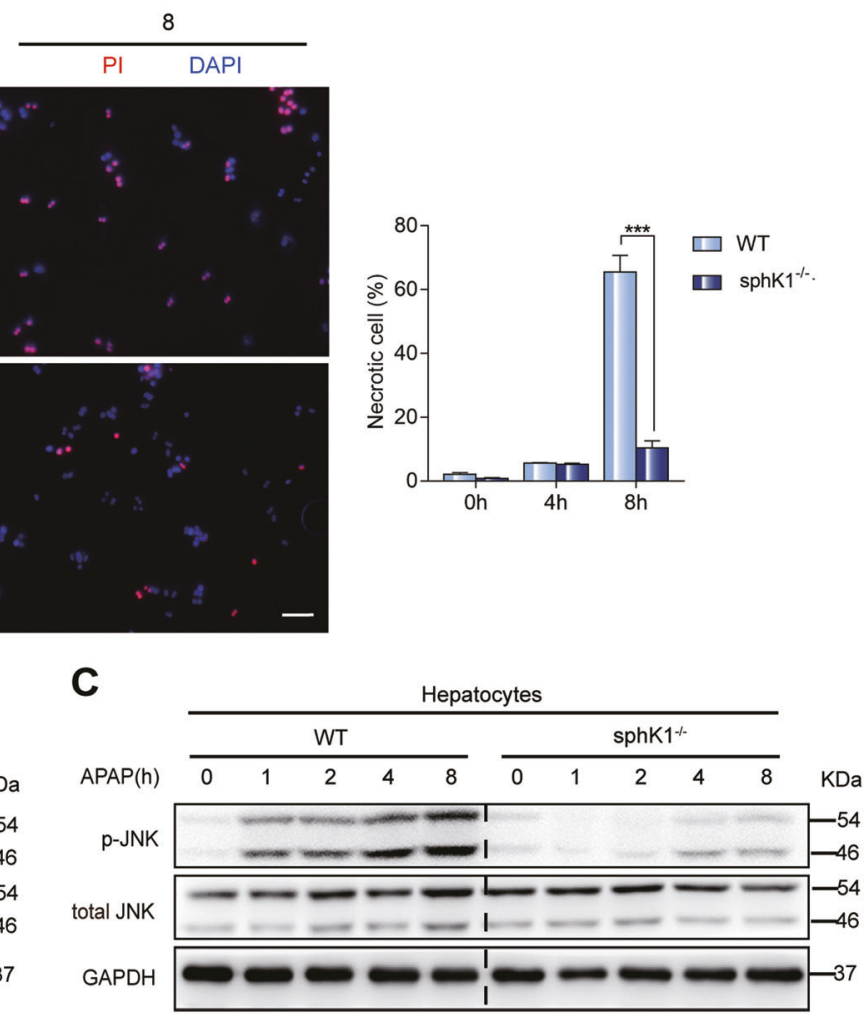

(200 mg/kg, i.p., $n=6-8$ per group), liver tissue was harvested at $0,2,4$, 8 , and $16 \mathrm{~h}$ post APAP treatment, and western blot was performed to determine the levels of phosphorylated and total JNK protein. $\mathbf{c}-\mathbf{d}$ primary hepatocytes from WT or $s p h K^{-1-}$ mice were treated with APAP $(10 \mathrm{~mm})$ for $0,1,2,4$, and $8 \mathrm{~h}$, and then $\mathbf{c}$ levels of phosphorylated and total JNK protein and $\mathbf{d}$ levels of BCL-2 and BAX proteins in total cell lysates of primary hepatocytes were analyzed by western blotting.

Together, these results indicate that SPHK1 is essential for the activation of multiple key signaling molecules involved in MPT.

\section{SPHK1 deficiency alleviates the expression of inflammatory genes by regulating histone deacetylases (HDACs)}

Upon APAP treatment, IL-6, TNF- $\alpha$, and IL- $1 \beta$ levels were significantly increased in supernatants derived from primary hepatocytes isolated from WT mice but not in those from $s p h \mathrm{Kl}^{-/-}$mice (Fig. 7a). In addition, mRNA 
A

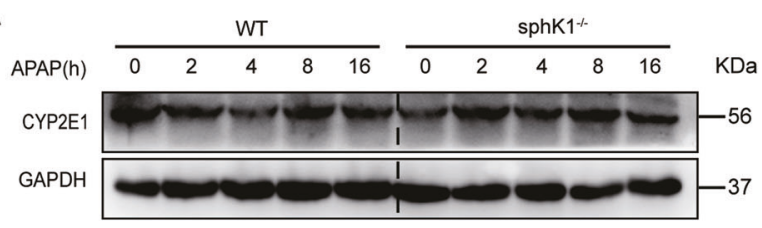

C

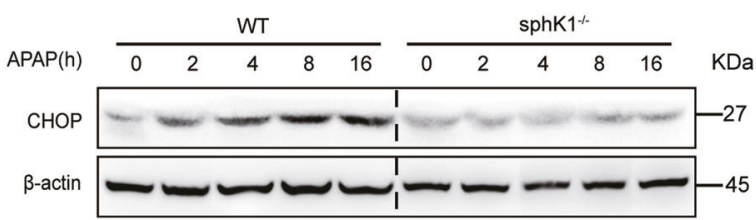

D

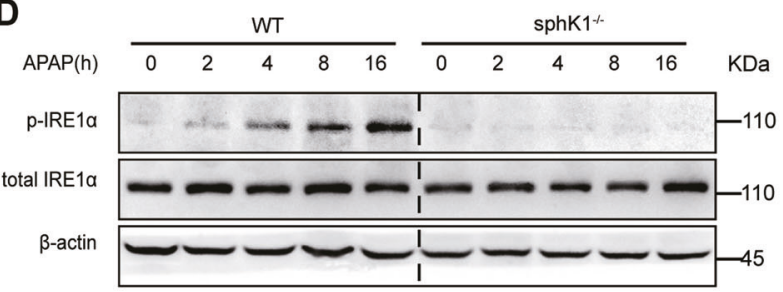

$\mathbf{F}$

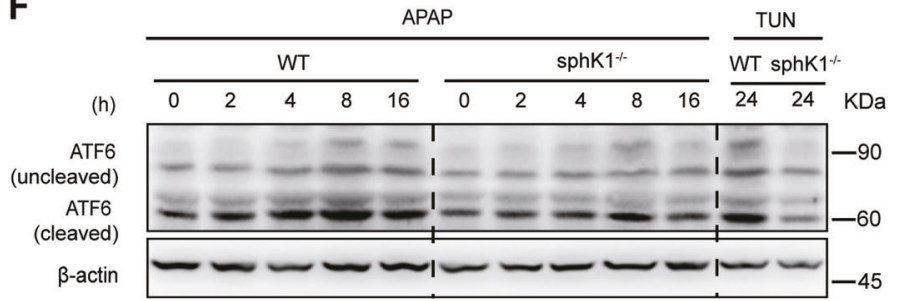

B

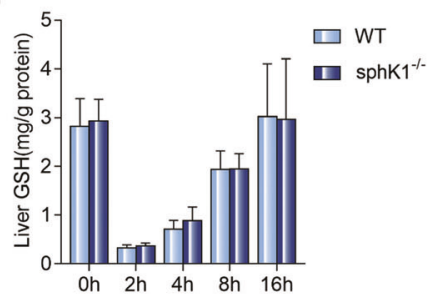

$\mathbf{E}$

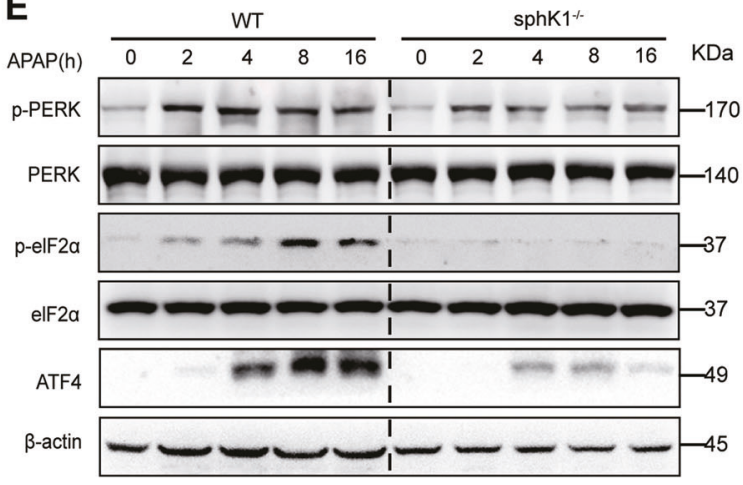

G
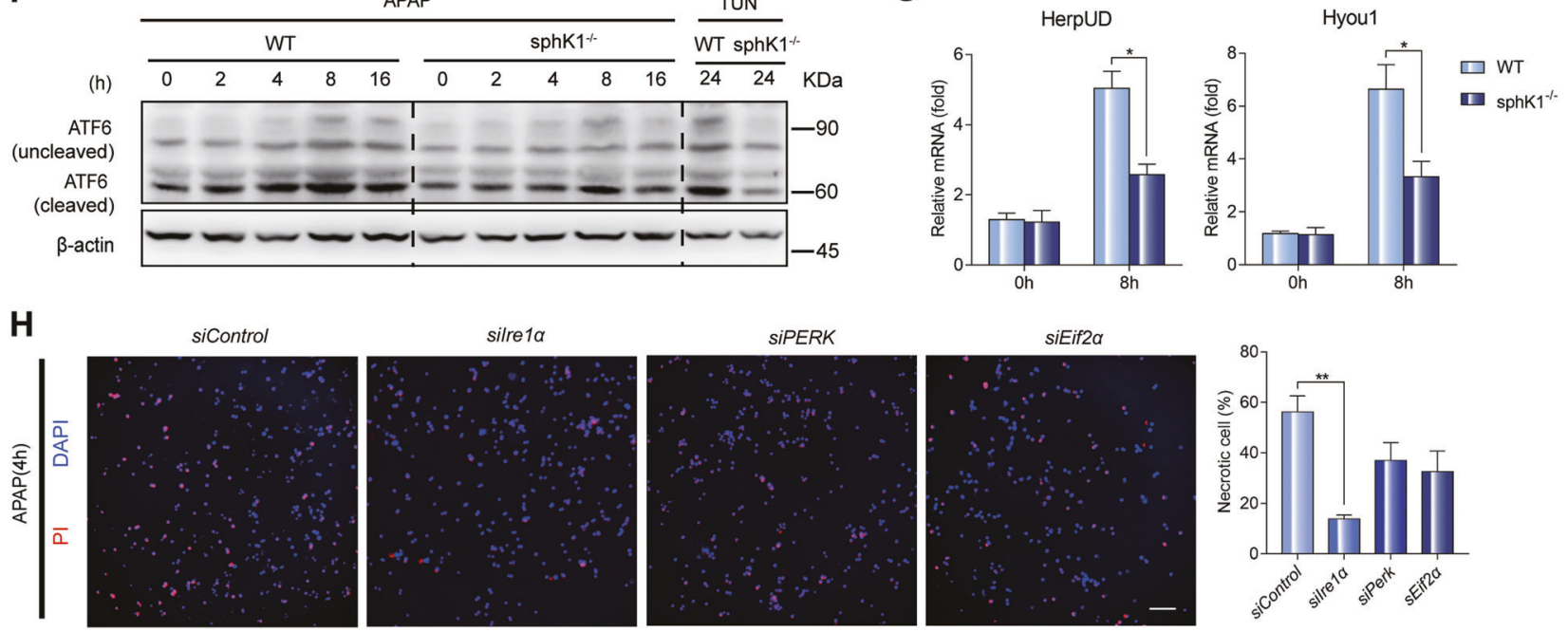

Fig. 5 SPHK1 deficiency alleviates ER stress during APAPinduced hepatotoxicity. a-b WT and $\operatorname{sphK1^{-/-}}$ mice were treated with APAP (200 mg/kg, i.p.), liver tissue was harvested at 0, 2, 4, 8, and $16 \mathrm{~h}$ post APAP treatment, and then a levels of CYP2E1 in the liver were determined by western blotting, and b levels of GSH in the liver were quantified using a GSH assay kit; data represent mean GSH \pm SEM. c-f WT and $s p h \mathrm{~K}^{-1-}$ mice were treated with APAP $(200 \mathrm{mg} / \mathrm{kg}$, i.p.), liver tissue was harvested at $0,2,4,8$, and $16 \mathrm{~h}$ post APAP treatment, and then $\mathbf{c}$ protein levels of CHOP, $\mathbf{d}$ levels of phosphorylated and total IRE1 $\alpha$, e levels of phosphorylated and total PERK-eIF2 $\alpha$ and ATF4 protein levels, and $\mathbf{f}$ levels of cleaved and uncleaved ATF6 in the liver

levels of Il-6, Tnf- $\alpha$, and $I l-1 \beta$ in $\operatorname{sphK1^{-/-}}$ hepatocytes were also significantly lower than those in WT hepatocytes (Fig. 7b). Mechanistically, when treated with APAP,

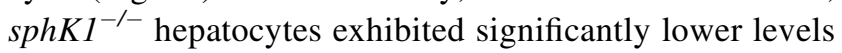
of phosphorylated and total TRAF2 (Fig. 7c), as well as lower levels of phosphorylated p65 (Fig. 7c) and tissue were determined by western blotting; liver lysates of tunicamycin

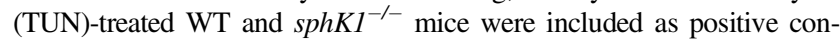
trols. g WT or $\operatorname{sphK1^{-1-}}$ mice were treated with APAP (200 mg/kg, i.p.), and then mRNA levels of HerpUD and Hyoul in liver tissues of WT and $\operatorname{sphK} 1^{-1-}$ mice at 0,8 , and $16 \mathrm{~h}$ after treatment with APAP were measured by quantitative real-time PCR. h Murine AML12 hepatocytes were transfected with siRNA for Ire $1 \alpha$, Perk, or Eif $2 \alpha$, or with control siRNA, and then cells were treated with APAP $(10 \mathrm{~mm})$; cell death was quantified with propidium iodide (PI) staining. Data represent the mean ratio of the number of necrotic cells to total cell number \pm SEM. $* P<0.05 ; * * P$ $<0.01$ using two-tailed unpaired Student's $t$ test.

phosphorylated p38 (Fig. S9) than did WT hepatocytes. Further, SPHK1 deficiency reduced APAP-induced phosphorylation of IKB, as well as the nuclear translocation of p65 (Fig. 7d).

We also examined whether SPHK1 deficiency affected any epigenetic phenotype in primary hepatocytes after 


\section{A}

$\operatorname{APAP}(\mathrm{h})$ 0
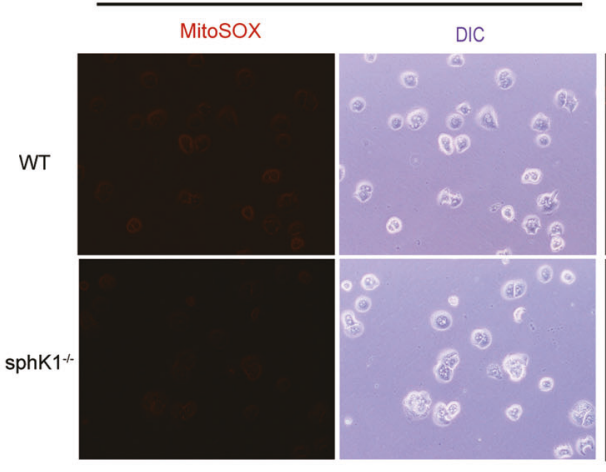

4

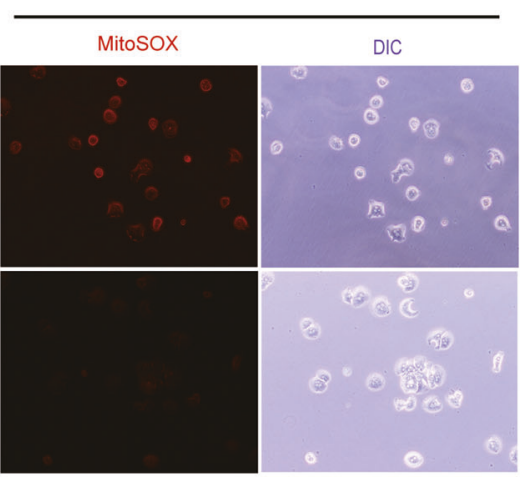

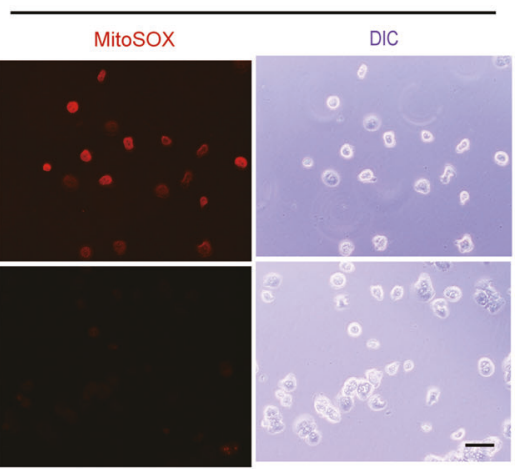

B

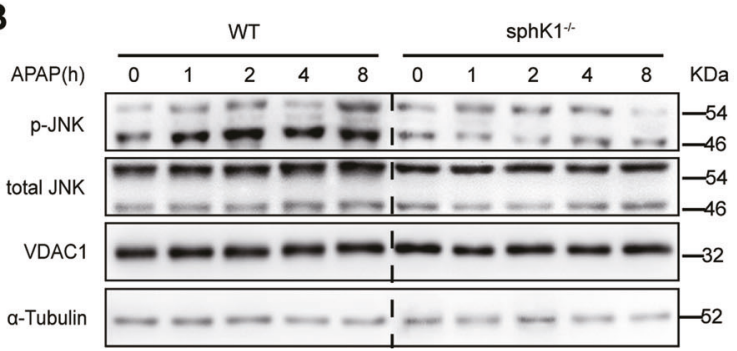

D

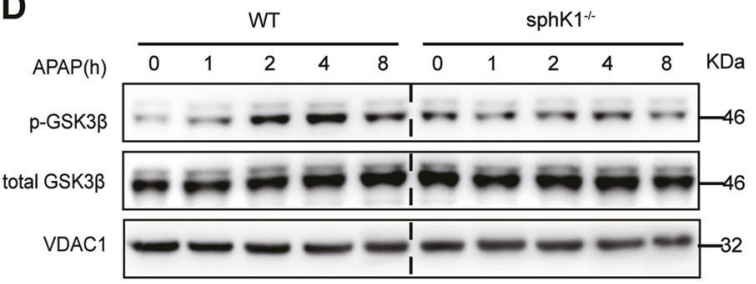

Fig. 6 APAP treatment alters mitochondrial permeability transition in primary hepatocytes from WT and $s p h K 1^{-1-}$ mice. a Primary hepatocytes from WT and $s p h \mathrm{~K}^{-/-}$mice were treated with $10 \mathrm{~mm} \mathrm{APAP}$ at 0,4 , and $8 \mathrm{~h}$, and then mitochondrial reactive oxygen species in primary hepatocytes were stained with MitoSOX Red, scale bar: $200 \mu \mathrm{m}$. b-d Primary hepatocytes from WT and $\operatorname{sphK1^{-1-}}$ mice

APAP exposure. There were no significant differences in methylated levels of $\mathrm{H} 3 \mathrm{~K} 4, \mathrm{H} 3 \mathrm{~K} 9$, and $\mathrm{H} 3 \mathrm{~K} 27$ between

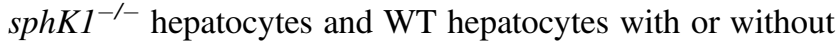
APAP treatment (Fig. S10a). Although the levels of H3K9ac were not significantly affected, the decreased level of H4K5ac was indeed reversed by SPHK1 deficiency (Fig. S10b). Interestingly, the increased protein (Fig. 7e) and mRNA (Fig. S11) levels of HDAC4, HDAC5, and HDAC7 were significantly lower in $s p h K^{-1-}$ hepatocytes following APAP treatment than in WT hepatocytes. The acetylation levels of p65 (Fig. 7f) and STAT1 (Fig. 7g) were significantly increased in $\operatorname{sphKl^{-/-}}$ hepatocytes treated with APAP, as compared with those in APAP-treated WT hepatocytes (Fig. 7f, g). In addition, SPHK1 deficiency significantly increased the interaction between acetylated p65 and STAT1 after APAP treatment (Fig. 7h), therefore inhibiting the transcription of proinflammatory genes.
C

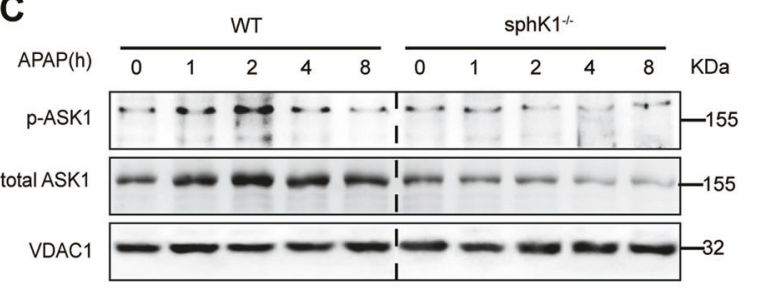

were treated with $10 \mathrm{~mm}$ APAP, and then mitochondrial lysates were isolated by commercial Mitochondria Isolation Kit. Levels of phosphorylated and total proteins of b JNK, $\mathbf{c}$ ASK1, and d GSK3 $\beta$ in mitochondrial lysates were analyzed by western blotting with VDAC1 as the internal loading control and $\alpha$-tubulin as the negative control.

\section{Exogenous S1P partially reverses the effects of SPHK1 deficiency}

APAP treatment slightly increased S1P levels but not significantly (Fig. S12a). However, in $s p h K 1^{-/-}$hepatocytes, exogenous S1P restored the activation of eIF2 $\alpha$ and ATF6 levels in response to APAP, but not the levels of CHOP or IRE1 $\alpha$ phosphorylation (Fig. 8a). In these cells, the activation of key MPT mediators, including JNK, ASK1, and GSK3 $\beta$, as well as TRAF2 was also restored by exogenous S1P (Fig. 8b), as was the phosphorylation of p65 and p38 (Fig. 8c). In addition, treatment with FTY720, an antagonist of S1P receptors, completely protected WT mice after treatment with APAP, as evidenced by the significantly reduced ALT and AST serum levels (Fig. 8d), the significantly decreased serum and mRNA levels of inflammatory cytokines (Fig. S12b-c), and the markedly increased survival rate after a lethal dose 

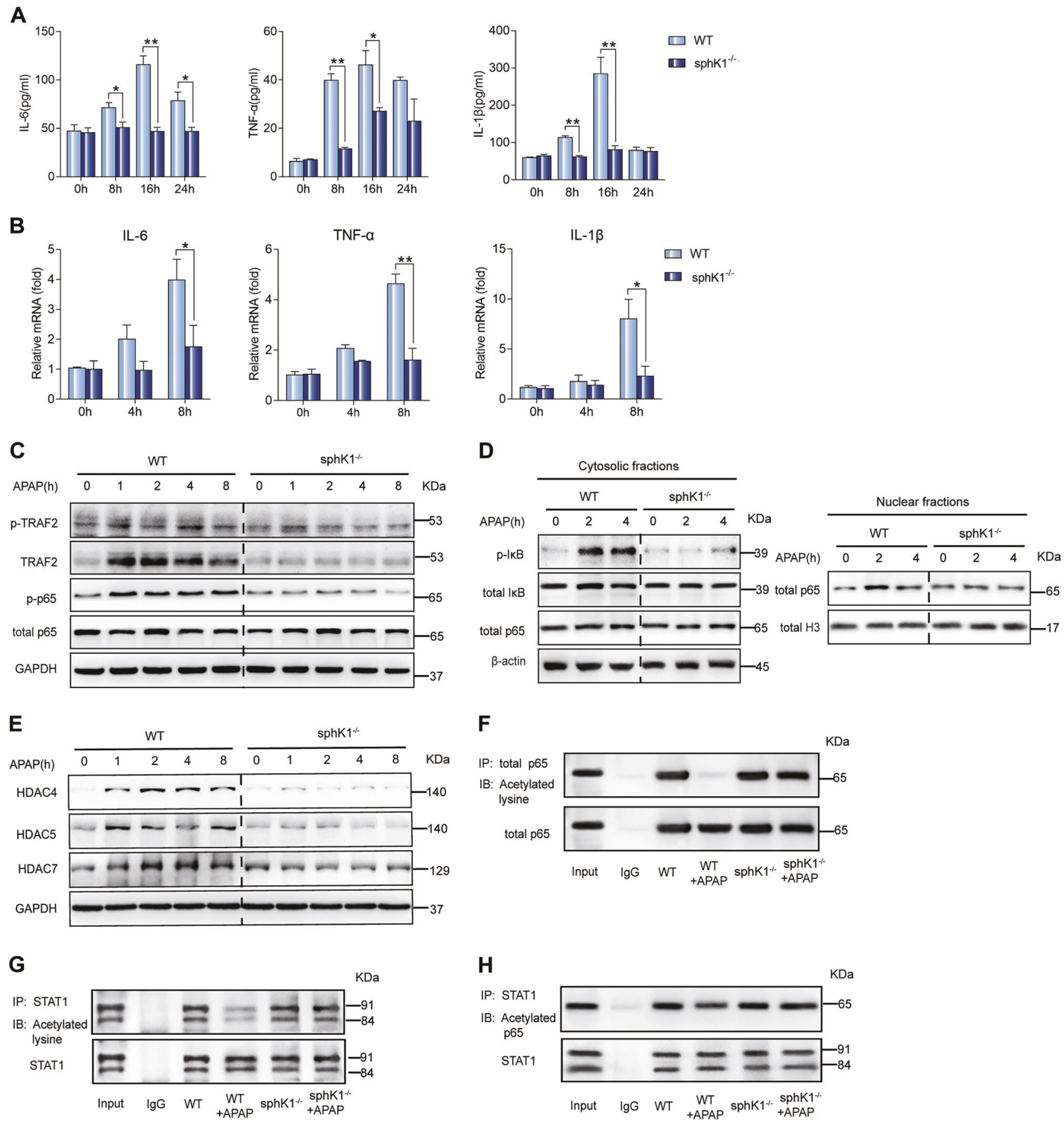

Fig. 7 APAP fails to induce inflammation in the absence of SPHK1. a Primary hepatocytes isolated from WT and $s p h K 1^{-1-}$ mice were treated with $10 \mathrm{~mm}$ APAP at various time points, and then levels of inflammatory cytokines IL- 6 , TNF- $\alpha$, and IL- $1 \beta$ in cultured hepatocyte supernatants were assayed by ELISA ( $n=6$ per group). b mRNA levels of inflammatory cytokines $\mathrm{Il}-6$, Tnf- $\alpha$, and $\mathrm{Il}-1 \beta$ in APAP-treated WT and $\operatorname{sphK} 1^{-1-}$ hepatocytes were quantified by real-time PCR ( $n=4-6$ per group). Data represent mean expression \pm SEM. $* P<0.05 ; * * P<0.01$ using two-tailed unpaired Student's $t$ test. c-e Primary hepatocytes isolated from WT and $\operatorname{sphK1^{-/-}}$ mice

were treated with $10 \mathrm{~mm}$ APAP at various time points. Levels of c phosphorylated and total protein of TRAF2 and p65 in total

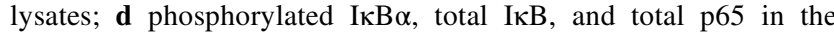
cytosolic fraction and p65 in the nuclear fraction; and e HDAC4, HDAC5, and HDAC7 in total lysates were determined by western blotting. $\mathbf{f}-\mathbf{h}$ Primary hepatocytes isolated from WT mice or $s p h K 1$

${ }^{-/}$mice were treated with APAP $(10 \mathrm{~mm}, 2 \mathrm{~h})$, and then the acetylation of $\mathbf{f}$ p 65 and $\mathbf{g}$ STAT1 as well as $\mathbf{h}$ the levels of interaction between acetylated p 65 and STAT1 in total lysates were analyzed by co-immunoprecipitation.

(700 mg/kg) of APAP (Fig. 8e). The fact that S1P was not able to promote the activation of IRE1 $\alpha$ and CHOP

led us to hypothesize that S1P-independent mechanisms are involved in activating these proteins. Further, 
A

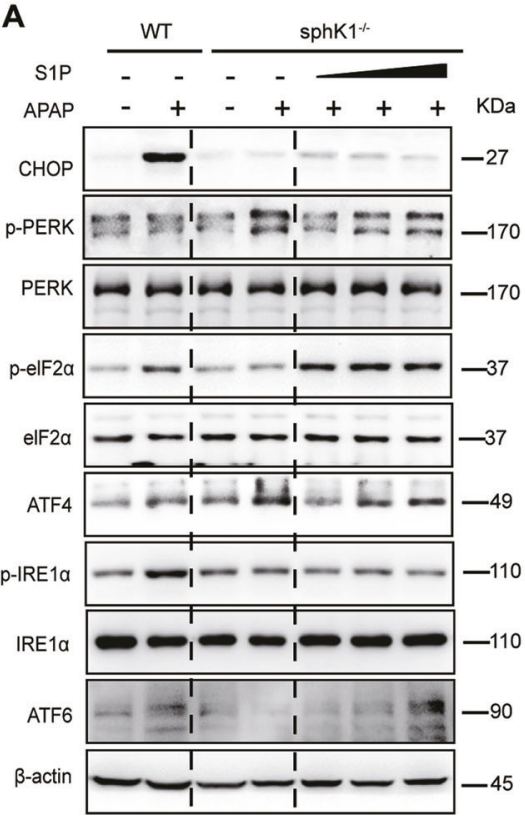

D
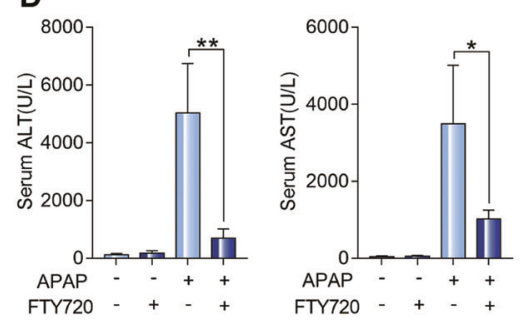

E

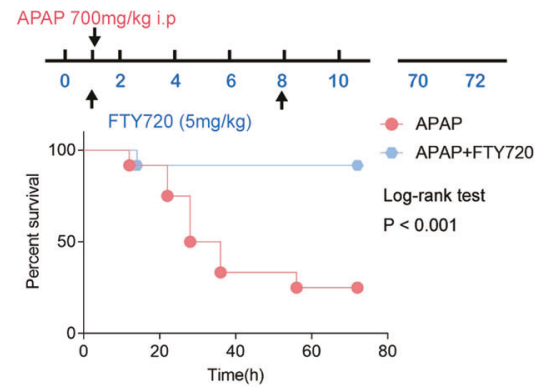

Fig. 8 Both S1P-dependent and -independent mechanisms are involved in APAP-induced acute liver hepatotoxicity. a-c Exogenous $\mathrm{S} 1 \mathrm{P}$ at various concentrations $(0.2,1.0$, and $5.0 \mu \mathrm{g} / \mathrm{ml})$ was supplemented in primary WT and $s p h K 1^{-1-}$ hepatocytes in culture. a Levels of phosphorylated PERK-eIF2 $\alpha$ and IRE1 $\alpha$, protein levels of CHOP, ATF4, and ATF6; b phosphorylation of JNK, ASK1, and GSK3 $\beta$; and $\mathbf{c}$ TRAF2, phosphorylated p65, and p38 were analyzed by western blotting. d WT mice were treated with APAP $(200 \mathrm{mg} / \mathrm{kg}$, i.p. $)$

through immunoprecipitation, we found that SPHK1 formed a complex with IRE1 $\alpha$ in vivo (Fig. S13a). In murine AML12 hepatocytes, Ire $1 \alpha$ siRNA transfection significantly reduced the phosphorylation of ASK1 and JNK (Fig. S13b). Figure 8i shows the schematic view of the SPHK1-mediated molecular signaling mechanism underlying APAP-induced hepatotoxicity.

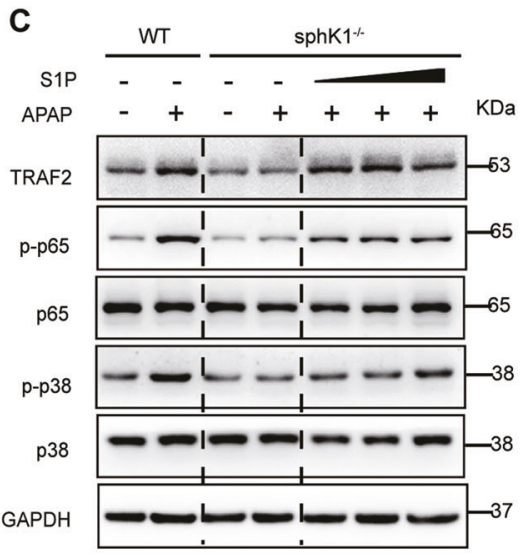

$\mathbf{F}$

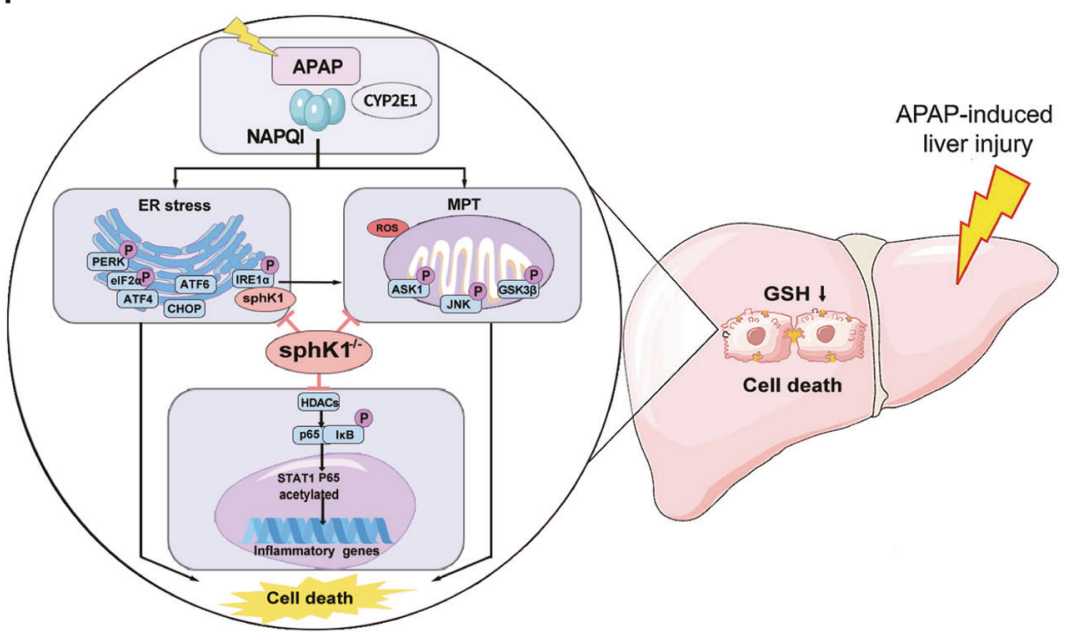

or with APAP and FTY720 (10 mg/kg, i.p., $n=6$ per group), sera were harvested $16 \mathrm{~h}$ post APAP treatment, and levels of ALT and AST in the control and FTY720-treated mice $(5 \mathrm{mg} / \mathrm{kg}$, i.p.) were measured. e Survival curve of WT mice treated with a lethal dose of APAP $(700 \mathrm{mg} / \mathrm{kg})$ and of WT mice treated with APAP and FTY720 $(5 \mathrm{mg} / \mathrm{kg}$, i.p., $n=12$ per group). f Schematic illustration of the putative signaling mechanism underlying APAP-induced hepatic cell death.

\section{Discussion}

Understanding the molecular mechanism of APAP-induced acute liver damage and hepatocyte death is fundamental to the design of new therapeutic strategies for the treatment of this type of liver injury. We now report that $s p h K 1^{-1-}$ mice exhibit substantially much less liver damage than WT 
control mice following an APAP overdose treatment, demonstrating that SPHK1 is essential in mediating APAPinduced acute liver injury. We further demonstrate that SPHK1 is not involved in the initial metabolism of APAP. Instead, SPHK1 promotes APAP-induced ER stress and mitochondrial dysfunction. ER stress precedes the occurrence of MPT in APAP-induced liver injury and other UPRtriggered cell death-related events $[29,30]$. Thus, our study highlights a previously undescribed mechanism by which SPHK1 promotes ER stress, thereby enhancing mitochondrial dysfunction and inflammation and ultimately causing APAP-induced hepatotoxicity.

$\mathrm{S} 1 \mathrm{P}$ is generated by the phosphorylation of sphingosine by two sphingosine kinase isoforms: SPHK1 and SPHK2. There is evidence showing that the expression of SPHK1 can be downregulated by $>70 \%$ with siRNA without affecting SPHK2 [31]. We also found that there were no significant changes in the protein levels of SPHK2 between

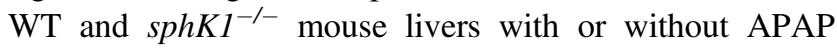
treatment. Despite the functional non-redundancy of these two enzymes in the production of S1P, our data showed that the levels of aminotransferases and inflammatory gene transcription in $s p h K 2^{-/}$mice were similar to those in WT mice when treated with APAP. Therefore, SPHK1 and SPHK2 cannot completely compensate for each other in mediating APAP-induced hepatotoxicity.

The SPHK1-S1P axis regulates an array of biological processes. For example, S1P not only engages extracellular receptors to regulate a series of downstream signals but also acts as a secondary messenger directly involved in intracellular signal transduction and regulation [32]. It has been reported that pharmacological inhibition or genetic deficiency of SPHKs results in the reduced activation of extracellular signal-regulated kinases in tumor cells [33]. Notably, S1P could efficiently stimulate the phosphorylation of many key molecules in different cellular signaling pathways. Therefore, the defective phosphorylation of these key molecules in sphKl-deficient hepatocytes may be attributable to the decreased production of S1P in these cells. The S1P receptor functional antagonist FTY720, an immunosuppressive agent currently used for the treatment of multiple sclerosis, has shown promise in reducing the intracellular levels of S1P [34]. In this study, FTY720 protected WT mice from APAP-induced liver injury and significantly enhanced survival rates of animals treated with $700 \mathrm{mg} / \mathrm{kg}$ APAP. However, exogenous S1P effectively reversed the effects of SPHK1 deficiency on TRAF2, eIF $2 \alpha$, and ATF6, but not those on CHOP and IRE1 $\alpha$, implying that additional S1P-independent mechanisms also exist. We previously reported that SPHK2 directly activates Fyn and downstream STAT3 to promote fibroblast activation and extracellular matrix production [35]. In hepatocytes, SPHK1 interacting with IRE1 $\alpha$ is one of the possible mechanisms of its activation of ER stress independent of S1P. In addition, the possibility that SPHK1 binds to other signaling molecules to promote ER stress or MPT directly or indirectly cannot be ruled out. Nevertheless, it is clear that SPHK1 deficiency prevents the activation of major signaling pathways that elicit ER stress in hepatocytes, which would otherwise trigger MPT, inflammatory responses, and ultimately hepatocyte necrosis.

The fact that SPHK1 deficiency dramatically alters gene transcription in the liver prompted us to hypothesize that epigenetic mechanisms are involved in this process. Interestingly, the levels of HDAC4, HDAC5, and HDAC7 were significantly reduced in $s p h K 1$-deficient hepatocytes treated with APAP. Although there was no significant difference in the levels of histone acetylation, this reduction is consistent with the fact that HDAC inhibitors can protect the liver from other inflammatory conditions [36, 37].

Recent studies have shown that HDACs and the acetylation of STAT1 and p65 play a vital role in the regulation of NF- $\mathrm{KB}$ transcriptional activity $[38,39]$. Here, we showed that following APAP treatment, the levels of acetylated p65 and STAT1 were significantly decreased in WT hepatocytes, but not in sphKl-deficient hepatocytes. Through co-immunoprecipitation, we also showed that SPHK1 deficiency promoted an interaction between STAT1 and p65, subsequently alleviating the transcription of inflammatory genes induced by APAP. Therefore, SPHK1 deficiency inhibits NF-KB transcriptional activity by maintaining p65 in its acetylated (inactive) state, which is normally regulated by HDACs, thereby repressing the expression of inflammatory cytokines.

In summary, our study reveals that SPHK1 in hepatocytes is a critical promotor in the initial phase of APAP-induced acute liver failure. We have unraveled a critical mechanism controlling APAP-induced acute liver injury that is mediated by SPHK1-dependent regulation of ER stress and MPT. Our data highlight SPHK1 as a potential therapeutic target against APAP-induced hepatotoxicity and may therefore open a new avenue to enable effective treatment of APAPinduced hepatotoxicity and its associated complications.

\section{Materials and methods}

\section{Mice}

$s p h K 1^{-/-}$mice, sphK2 $2^{-/-}$mice, and wild-type (WT) control mice, on a C57BL/6 J background and aged 8-10 weeks, were obtained from the Jackson Laboratory (Bar Harbor, ME, USA), and were bred and housed at the Animal Care Facility of Nanjing Medical University under specific pathogen-free conditions with ad libitum access to a standard laboratory diet and water. When used in experiments, male mice at $8-10$ weeks old were fasted for $12 \mathrm{~h}$ overnight 
and then injected intraperitoneally (i.p.) with APAP (SigmaAldrich, St. Louis, MO) dissolved in warm phosphatebuffered saline [40]. Prior to injection, animals were fasted to improve the uniformity of drug administration and to reduce APAP glucuronidation. Following injection, animals were fed ad libitum until time of killing [41]. All experimental protocols were approved by the Laboratory Animal Care and Use Committee at Nanjing Medical University (NJMUAC No. 053/2016).

\section{Bone marrow transplantation}

In brief, donor mice were killed, and bone marrow cells were harvested from the humerus and femur. Recipient mice ( 8 weeks old) were pretreated with antibiotics (Sangon Biotech, Shanghai, China) and lethally irradiated with two doses of $5 \mathrm{~Gy}$ at a $2-3 \mathrm{~h}$ interval. Following this, $10^{7}$ donor bone marrow cells were transferred intravenously through the tail vein into each of the recipients. Donor cells were allowed to engraft for 8 weeks. Untransplanted $\operatorname{sphK1^{-1-}}$ and WT mice were used as controls.

\section{Hepatotoxicity assessment}

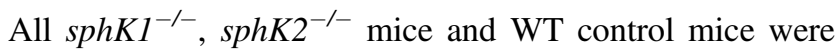
humanely killed, and sera were collected at 8,16 , and $24 \mathrm{~h}$ post APAP treatment. ALT and AST activities were quantified using commercially available kits (Sigma-Aldrich, St. Louis, MO, USA) according to the manufacturer's protocols.

\section{Cell death determination}

WT primary hepatocytes or $s p h \mathrm{Kl}^{-/-}$primary hepatocytes were seeded in six-well plates, exposed to $10 \mathrm{~mm}$ APAP and analyzed at 0,4 , and $8 \mathrm{~h}$ to determine cell death and morphological changes by staining for $30 \mathrm{~min}$ with $1 \mu \mathrm{g} / \mathrm{mL}$ propidium iodide (Sigma-Aldrich). Fluorescence images were obtained in RGB at 32 bits/channel using an Olympus IX-73 microscope (Olympus, Tokyo, Japan).

\section{Co-immunoprecipitation}

Tissue or cell lysates were incubated overnight at $4{ }^{\circ} \mathrm{C}$ with $1 \mu \mathrm{g}$ of anti-SPHK1, anti-STAT1, or anti-p65 antibodies (CST, Boston, MA, USA). A 40- $\mu \mathrm{L}$ volume of protein A/G agarose beads (Roche, Mannheim, Germany) was then added to lysates and incubated for 4-6 h. The agarose beads were pelleted by a brief centrifugation $(900 \mathrm{~g}, 10 \mathrm{~min})$ and then washed three times with lysis buffer. Immunoprecipitated proteins were eluted by boiling in SDS buffer for $10 \mathrm{~min}$. The protein levels of IRE1 $\alpha$ and acetylation of STAT1 and p65 were analyzed by western blotting.
Measurement of reactive oxygen species (ROS)

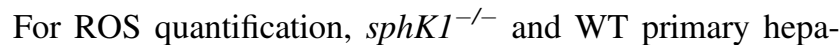
tocytes were treated with $10 \mathrm{~mm}$ APAP for 4 and $8 \mathrm{~h}$ and loaded with MitoSOX Red (Invitrogen, Carlsbad, CA, USA). ROS levels were determined by measuring the fluorescence excitation/emission spectra at $485 / 520 \mathrm{~nm}$ under a fluorescence microscope (Olympus).

\section{Hepatic GSH measurement}

Hepatic GSH levels were measured in whole-liver homogenates (15-25 mg of liver tissue) using a glutathione assay kit (Cayman, Ann Arbor, MI, USA) according to the manufacturer's instructions.

\section{Statistical analysis}

All data are expressed as the means \pm SEM. Data were compared using an unpaired two-tailed Student's $t$ test or a one-way analysis of variance and Bonferroni multiple comparisons test with SPSS software (version 17.0 for Windows, IBM Inc, Chicago, IL, USA). $P<0.05, P<0.01$, and $P<0.001$ were considered significant $(*)$, very significant $(* *)$, and highly significant $(* * *)$, respectively.

Additional methodological details are provided in the Supplementary Materials.

Acknowledgements This work was supported by grants from the National Key Research and Development Program of China (Grant No. 2016YFF0101400), National Natural Science Foundation of China (Grant No. 81571614, 81871241), and Jiangsu Provincial Innovation Team Program Foundation.

Author contributions LjL and HZ conceptualized and designed the study; LjL, HW, JZ, YS, and FW performed the experiments; LjL performed the statistical analysis; LjL, HW, and $\mathrm{HZ}$ drafted the manuscript; SW, LS, QY, MS, LxL, and HZ revised and approved the final version of the manuscript. All authors read and approved the manuscript.

\section{Compliance with ethical standards}

Conflict of interest The authors declare that they have no conflict of interest.

Publisher's note Springer Nature remains neutral with regard to jurisdictional claims in published maps and institutional affiliations.

\section{References}

1. Ostapowicz G, Fontana RJ, Schiodt FV, Larson A, Davern TJ, $\mathrm{Han} \mathrm{SH}$, et al. Results of a prospective study of acute liver failure at 17 tertiary care centers in the United States. Ann Intern Med. 2002;137:947-54.

2. Schilling A, Corey R, Leonard M, Eghtesad B. Acetaminophen: old drug, new warnings. Clevel Clin J Med. 2010;77:19-27. 
3. Lee WM. Acetaminophen toxicity: changing perceptions on a social/medical issue. Hepatology. 2007;46:966-70.

4. Dahlin DC, Miwa GT, Lu AY, Nelson SD. N-acetyl-pbenzoquinone imine: a cytochrome P-450-mediated oxidation product of acetaminophen. Proc Natl Acad Sci USA. 1984;81:1327-31.

5. Coles B, Wilson I, Wardman P, Hinson JA, Nelson SD, Ketterer B. The spontaneous and enzymatic reaction of N-acetyl-pbenzoquinonimine with glutathione: a stopped-flow kinetic study. Arch Biochem Biophys. 1988;264:253-60.

6. Hinson JA, Pohl LR, Monks TJ, Gillette JR. Acetaminopheninduced hepatotoxicity. Life Sci. 1981;29:107-16.

7. Lawson JA, Farhood A, Hopper RD, Bajt ML, Jaeschke H. The hepatic inflammatory response after acetaminophen overdose: role of neutrophils. Toxicol Sci. 2000;54:509-16.

8. Williams CD, Bajt ML, Farhood A, Jaeschke H. Acetaminopheninduced hepatic neutrophil accumulation and inflammatory liver injury in CD18-deficient mice. Liver Int. 2010;30:1280-92.

9. Tacke F. Targeting hepatic macrophages to treat liver diseases. J Hepatol. 2017;66:1300-12.

10. Uzi D, Barda L, Scaiewicz V, Mills M, Mueller T, GonzalezRodriguez A, et al. CHOP is a critical regulator of acetaminopheninduced hepatotoxicity. J Hepatol. 2013;59:495-503.

11. Dara L, Ji C, Kaplowitz N. The contribution of endoplasmic reticulum stress to liver diseases. Hepatology. 2011;53:1752-63.

12. Zhang K, Kaufman RJ. From endoplasmic-reticulum stress to the inflammatory response. Nature. 2008;454:455-62.

13. Wang M, Kaufman RJ. Protein misfolding in the endoplasmic reticulum as a conduit to human disease. Nature. 2016;529:326-35.

14. Malhi H, Kaufman RJ. Endoplasmic reticulum stress in liver disease. J Hepatol. 2011;54:795-809.

15. Lisbona F, Rojas-Rivera D, Thielen P, Zamorano S, Todd D, Martinon F, et al. BAX inhibitor-1 is a negative regulator of the ER stress sensor IRE1alpha. Mol Cell. 2009;33:679-91.

16. Urano F, Wang X, Bertolotti A, Zhang Y, Chung P, Harding HP, et al. Coupling of stress in the ER to activation of JNK protein kinases by transmembrane protein kinase IRE1. Science. 2000;287:664-6.

17. Rothe M, Sarma V, Dixit VM, Goeddel DV. TRAF2-mediated activation of NF-kappa B by TNF receptor 2 and CD40. Science. 1995;269:1424-7.

18. Seki E, Brenner DA, Karin M. A liver full of JNK: signaling in regulation of cell function and disease pathogenesis, and clinical approaches. Gastroenterology. 2012;143:307-20.

19. Hanawa N, Shinohara M, Saberi B, Gaarde WA, Han D, Kaplowitz N. Role of JNK translocation to mitochondria leading to inhibition of mitochondria bioenergetics in acetaminopheninduced liver injury. J Biol Chem. 2008;283:13565-77.

20. Han D, Dara L, Win S, Than TA, Yuan L, Abbasi SQ, et al. Regulation of drug-induced liver injury by signal transduction pathways: critical role of mitochondria. Trends Pharm Sci. 2013;34:243-53.

21. Win S, Than TA, Zhang J, Oo C, Min RWM, Kaplowitz N. New insights into the role and mechanism of c-Jun-N-terminal kinase signaling in the pathobiology of liver diseases. Hepatology. 2018;67:2013-24.

22. Nakagawa H, Maeda S, Hikiba Y, Ohmae T, Shibata W, Yanai A, et al. Deletion of apoptosis signal-regulating kinase 1 attenuates acetaminophen-induced liver injury by inhibiting c-Jun $\mathrm{N}$-terminal kinase activation. Gastroenterology. 2008;135:1311-21.

23. Chang $\mathrm{CH}$, Randolph GJ. Sphingosine-1-phosphate as the lymphocyte's ticket to ride and survive. Dev Cell. 2017;41:576-8.

24. Rivera J, Proia RL, Olivera A. The alliance of sphingosine-1phosphate and its receptors in immunity. Nat Rev Immunol. 2008;8:753-63.
25. Maceyka M, Harikumar KB, Milstien S, Spiegel S. Sphingosine1-phosphate signaling and its role in disease. Trends Cell Biol. 2012;22:50-60.

26. Spiegel S, Milstien S. The outs and the ins of sphingosine-1phosphate in immunity. Nat Rev Immunol. 2011;11:403-15.

27. Liu H, Ma Y, He HW, Zhao WL, Shao RG. SPHK1 (sphingosine kinase 1) induces epithelial-mesenchymal transition by promoting the autophagy-linked lysosomal degradation of CDH1/E-cadherin in hepatoma cells. Autophagy. 2017;13:900-13.

28. Yang L, Yue S, Yang L, Liu X, Han Z, Zhang Y, et al. Sphingosine kinase/sphingosine 1-phosphate ( $\mathrm{S} 1 \mathrm{P}) / \mathrm{S} 1 \mathrm{P}$ receptor axis is involved in liver fibrosis- associated angiogenesis. J Hepatol. 2013;59:114-23.

29. Win S, Than TA, Fernandez-Checa JC, Kaplowitz N. JNK interaction with Sab mediates ER stress induced inhibition of mitochondrial respiration and cell death. Cell Death Dis. 2014;5: e989.

30. Deniaud A, Sharaf el dein O, Maillier E, Poncet D, Kroemer G, Lemaire C, et al. Endoplasmic reticulum stress induces calciumdependent permeability transition, mitochondrial outer membrane permeabilization and apoptosis. Oncogene. 2008;27:285-99.

31. Alvarez SE, Harikumar KB, Hait NC, Allegood J, Strub GM, Kim EY, et al. Sphingosine-1-phosphate is a missing cofactor for the E3 ubiquitin ligase TRAF2. Nature. 2010;465:1084-8.

32. Vienken H, Mabrouki N, Grabau K, Claas RF, Rudowski A, Schomel N, et al. Characterization of cholesterol homeostasis in sphingosine-1-phosphate lyase-deficient fibroblasts reveals a Niemann-Pick disease type C-like phenotype with enhanced lysosomal $\mathrm{Ca}(2+)$ storage. Sci Rep. 2017;7:43575.

33. Zhang H, Li W, Sun S, Yu S, Zhang M, Zou F. Inhibition of sphingosine kinase 1 suppresses proliferation of glioma cells under hypoxia by attenuating activity of extracellular signalregulated kinase. Cell Prolif. 2012;45:167-75.

34. Lee SY, Hong IK, Kim BR, Shim SM, Sung Lee J, Lee HY, et al. Activation of sphingosine kinase 2 by endoplasmic reticulum stress ameliorates hepatic steatosis and insulin resistance in mice. Hepatology. 2015;62:135-46.

35. Zhu X, Shi D, Cao K, Ru D, Ren J, Rao Z, et al. Sphingosine kinase 2 cooperating with Fyn promotes kidney fibroblast activation and fibrosis via STAT3 and AKT. Biochim Biophys Acta Mol Basis Dis. 2018;1864:3824-36.

36. Ferrari A, Fiorino E, Longo R, Barilla S, Mitro N, Cermenati G, et al. Attenuation of diet-induced obesity and induction of white fat browning with a chemical inhibitor of histone deacetylases. Int J Obes. 2017;41:289-98.

37. Lorenzo Pisarello M, Masyuk TV, Gradilone SA, Masyuk AI, Ding JF, Lee PY, et al. Combination of a histone deacetylase 6 inhibitor and a somatostatin receptor agonist synergistically reduces hepatorenal cystogenesis in an animal model of polycystic liver disease. Am J Pathol. 2018;188:981-94.

38. Chen S, Ye J, Chen X, Shi J, Wu W, Lin W, et al. Valproic acid attenuates traumatic spinal cord injury-induced inflammation via STAT1 and NF-kappaB pathway dependent of HDAC3. J Neuroinflammation. 2018;15:150.

39. Kumar P, Gogulamudi VR, Periasamy R, Raghavaraju G, Subramanian U, Pandey KN. Inhibition of HDAC enhances STAT acetylation, blocks NF-kappaB, and suppresses the renal inflammation and fibrosis in Npr1 haplotype male mice. Am J Physiol. 2017;313:F781-F795.

40. Kopec AK, Joshi N, Cline-Fedewa H, Wojcicki AV, Ray JL, Sullivan BP, et al. Fibrin(ogen) drives repair after acetaminopheninduced liver injury via leukocyte alphaMbeta2 integrindependent upregulation of Mmp12. J Hepatol. 2017;66:787-97.

41. Price VF, Jollow DJ. Effect of glucose and gluconeogenic substrates on fasting-induced suppression of acetaminophen glucuronidation in the rat. Biochem Pharm. 1989;38:289-97. 